\title{
ACQUIRING DESIGN LANGUAGE: CREATIVITY OF EXPERIMENTAL INK SEMANTICS CONVERSION IN DESIGN EDUCATION
}

\author{
Bo DU1, 2, Yukari NAGAI ${ }^{1}$, Eunyoung KIM${ }^{1}$, Takaya YUIZONO ${ }^{1}$ and Manqian WANG ${ }^{2}$ \\ ${ }^{1}$ Knowledge Science, Japan Advanced Institute of Science and Technology \\ ${ }^{2}$ National Demonstration Centre for Experimental Fashion Design and Engineering \\ Education, Dalian Polytechnic University
}

\begin{abstract}
Design requires a heterogeneous mixture of linguistics and culture and is a tool for visual communication [1]. The charm of language is reflected mainly in the accuracy and unique way with which information is disseminated. To acquire competencies with a design language is a process to seek information about creative thinking in practical courses. The ability to develop students' innovation in such a practical process is the most important component of art and design education. Ink, as an extension tool of design language development, provides great creativity and flexibility, which allows for the adequate preservation of the emotions derived from the designer's visual and dynamic experiences and for quick and easy recording of ideas. The goal of this research is to propose a method of curriculum design based on the theory and practice of oriental ink semantics, to demonstrate the process of innovation thinking practice and to showcase the possibilities of water-ink art in acquiring design language educational activities. The application of the concept of experimental water-ink semantics into the basic education of design has led to the conversion between the intrinsic value and design thinking of water-ink art, which leads to innovation in the process of creative design education. Through the design language conveying cultural elements, the design works resonate more easily. Thus, the cycle of creativity and design education is perpetuated.
\end{abstract}

\section{Keywords: Creativity practice, ink semantic, design language, conversion}

\section{INTRODUCTION}

The implementation process of designing basic education is a kind of learning that shapes students' creative ability through practical teaching interaction and communication. Design works can convey culture, art, technology, and professionalism and are an intermediate medium for communication between designers and viewers. Creativity is the process of creating new things and the phenomena of valuable things [2]. It is a comprehensive ability unique to human beings and is the main goal of artistic practice. It is composed of complex and multifactor comprehensive optimisation of knowledge, intelligence, ability, and excellent character quality. Cultivating and exerting creativity to convey the cultural spirit in the process of designing basic teaching practices has become an important development trend in today's design education [3].

Water-ink is not only a simple painting tool, but also a distinctive oriental cultural symbol. It contains the spirit of traditional culture and is an artistic style with a profound national cultural foundation [4]. With the development of the times, water-ink can achieve adaptive changes to meet people's current spiritual needs. Experimental ink is properly executed unique and non-repeatable ink element creativity. Ink can be used in the field of cultivating creativity design education as it is easy to operate and extremely malleable. For the observational study of objective objects to the artistic design education practice of imaginary abstract language acquisition, to obtain ink design language is an effective method and means. Interpreting the code of ink combined with design in the practical thinking process of designing basic education not only inherits traditional culture, but also expands the design ideas and methods and contributes to the formation of the art design language form. 
This research is based on how to obtain ink language with oriental characteristics in the practice of creativity, and it uses the design method and design process to solve the problem in the process of an actual conversion application. In the process of theoretical and practical research, this study proposed and solved the following. Q1: How can one obtain aesthetically a perception language in the practice of experimental ink tools? Q2: How can one realise a conversion application design that conveys the ink language and fashion a creative design practice? Presenting the practice process can lead to a better understanding of the generation of good design products and achieve a higher level of creativity.

\section{LITERATURE REVIEW}

From the perspective of the composition of painting tools, ink painting is a combination of water, ink, and a brush, a form of painting on rice paper (Xuan paper, a high-quality paper made in Xuancheng City, Anhui Province). First, the ink element has a "formal beauty" on the pen. Second, the artistic charm of ink elements is reflected in the use of ink. Moreover, because of the different ratio of water to ink, dry and wet changes are formed, thereby forming a distinct layered black-and-white grey-stage level with rich content.

\subsection{Concept of Language Elements: Pen and Ink}

The "pen" is a tool for the formation of ink language, referring to the brushwork, which emphasises how the pen works. The pen moves and changes to form lines and images on the paper, including the cultivation of calligraphy and the unique mastery practice of brush and ink. A plump vertical pen tip, vigorous and powerful reverse pen, and sharp use of the true sideways of the pen are all language elements that portray the objective world. The way to move the pen is to "write the image" and "draw the hieroglyphics" [5]. In the "ink bamboo" of the Song Dynasty painter Wen Tong, he used the pen writing method to paint the bamboo. Huai Su, a calligrapher of the Tang Dynasty, used a snakelike brushstroke in his crazy grass calligraphy works.

"Ink" refers to the ink method and technique - that is, how to use the different ratio of ink and water to express the art. In the Five Dynasties period, Jing Hao, in his important theoretical work The Brush Law, clearly stated: "The person using the brush can extend the application according to the rules of the pen, and in the depiction of the object texture and image, it is like real flight and movement." It can be seen that, in the concept of ink and pen, the ink refers to a kind of grasp of the ink's shade and thinness. Pen refers to the speed, skill, and method of the painter. The connotation of pen and ink reflects the different cultivation, temperament, aesthetic orientation, and personality of different people, which can be reflected in the pen and ink of the works. This is the most important value between the inner spiritual level and the ink language.

\subsection{Concept of Language Elements: Ink and Colour}

The ink has a strong subjective ability to express the dry and wet emotions and colours. Chinese painting advocates "simple" is also "plain," and this is reflected in the colour discipline, that is, "plain colour," black and white, which is the colour language of ink (Figure 1). Oriental art sums up the colourful colours in nature into endless changes between black and white. As shown in Figure 1-A, the catfish painted by Qi Baishi, a modern ink master, has reached the unity of pen, ink, and colour from artistic expression. The change of ink colour from the head to the tail is also a generalisation of the object. Ink is the colour, which means a change in the density of ink in water that is the cause of the various colours. The colour can be replaced by multilevel ink colour. Zhang Yanyuan, Tang Dynasty, in his record of the famous paintings of the past dynasties, The Story of the Paintings, said that: "ink is divided into five colours." The so-called "five colours" refer to coke, thick, heavy, light, and clear (Figure 1-B). It is the change of water that gives ink an endless grey tone to express life (Figure 1-C). The five inks of black, cyan, red, yellow, and white correspond to the brightness of different inks (Figure 1-D). It is like removing colour from a colour photo and turning it into a black-and-white photo. The brightness of the colour is unchanged, and the level of detail of the picture is not reduced.

Ink colour has the characteristics of purity and richness. According to the different ratios of ink and water, the ink produces different colours. If the colour is strong, the performance is real; if the colour is light, the performance is illusory. This is a rich change made by pursuing a single colour. Shown in the lotus flower paintings in Figure 1-E is the work of the painter "Bada Shanren" (real name Zhu Da) in the early Qing Dynasty. It is his design and creation of the summary of the real objects in the natural world using rich ink and colour. The ink colour refinement and correspondence is the true 
landscape of Figure 1-F. In short, the use of traditional ink painting and colour language innovation in design must grasp the spirit of ink and water, infiltrating the modern sense in the quaint atmosphere, so that simple and plain can touch people's hearts.

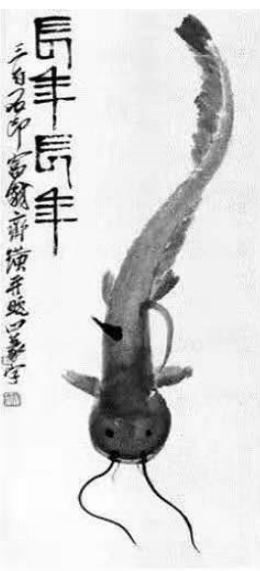

A

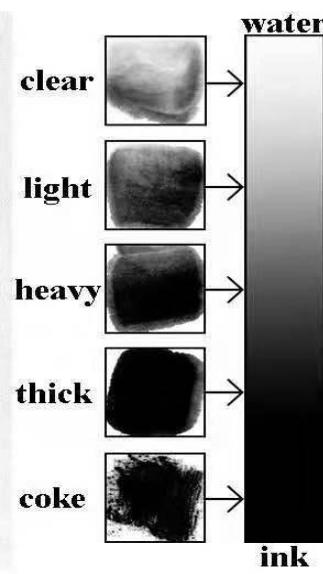

B

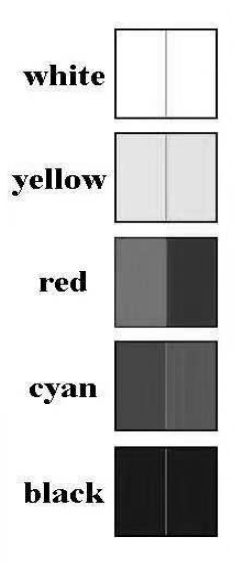

D

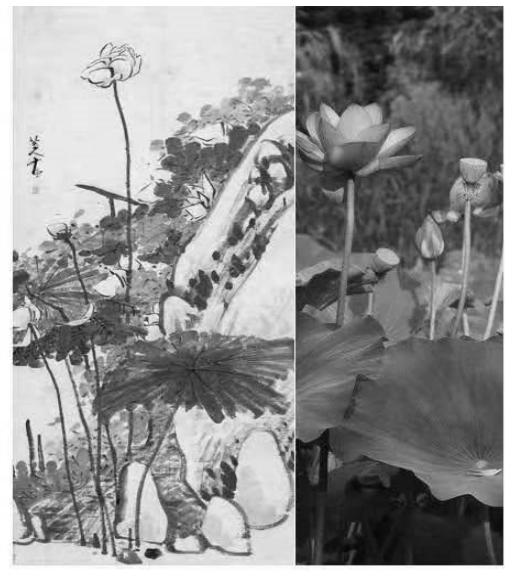

$\mathbf{E}$

$\mathbf{F}$

Figure 1. Highly generalised nature: ink and colour

\section{PRACTICE EXPERIMENT INK}

At present, at the National Demonstration Centre for Experimental Fashion Design and Engineering Education, Dalian Polytechnic University, the undergraduate design major of the School of Fashion includes fashion design engineering and apparel design. The professional direction includes character modelling, apparel product design, display design, and so on. In the design of the basic course construction, the ink and wash language are taken as the research object, and, through the interaction of practice, disciplines are developed to construct the intersection of the specific relationship between design language and product design [6]. The practice process consists of three parts: cognitive language exploration, practice development of ink semantics, and conversion in design (Figure 2).

\subsection{Cognitive Language Exploration}

The truth of nature is not the same as the truth of art. The truth of art makes it necessary to discover and create. The sensitivity of observation is a skill that can be improved through practice and can form an illusory subjective image in the brain. Consciously expressed by skilled hands and design thinking, both the ink and the body should be embodied in the picture, as well as the subjective thoughts of the designer.

- Through the deconstruction of artistic language, the ink language is used to describe the mountains, landscapes, plants, flowers, and creatures in nature.

- In the process of creativity practice, one also studies the likeness and dissimilarity, image and non-image, to forms subjective memory storage.

- Creators can freely use the theme of ink language based on subjective thinking, free from the constraints of images and technology, and support divergent thinking, which requires rich emotional guidance to create meaningful forms.

Therefore, saying "ink and wash" is not only a language method but also a spiritual carrier that can sharply inspire the emotional meaning of the creator and convey the creator's aesthetic judgment.

\subsection{Practice of Ink Semantics}

The acquisition process of the design language is to find the inner part of the emotions through the visualisation, and the practice of using ink is a static language description to present dynamic visual emotions. Ink language can illustrate the richness while, at the same time, taking into account the shape of the image, and it can use the chromaticity of the ink to weaken the shape to highlight the level of performance. The choices in the practice process also make the language unique.

- The process of practice is instructive, and it is a visualisation of thinking space. Focusing on cultivating imagination, it comes from the students' different understanding and preference for ink and water, depending on the personality characteristics of the students [7]. 
- The ductility of the paper carries the artistic effect of the ink, and the ink that smudges on the paper and the changes of the pen tip constitute the storage of the ink language memory. Ink language can transform the image into a form of ink, as well as the ability to organise and process artistic images.

- The construction of personal lateral thinking and vertical knowledge and emotional, visual, and dynamic experiences are integrated into the practice of ink and wash. With black and white, as the two poles of chromaticity, brightness is relatively unchanged. Only grey has a very broad performance space. The self-characteristics of grey colour are the result of a large level of change between water and ink and the reconciliation of the expressed objects. Experimental ink painting also achieves the training of "high-grade grey."

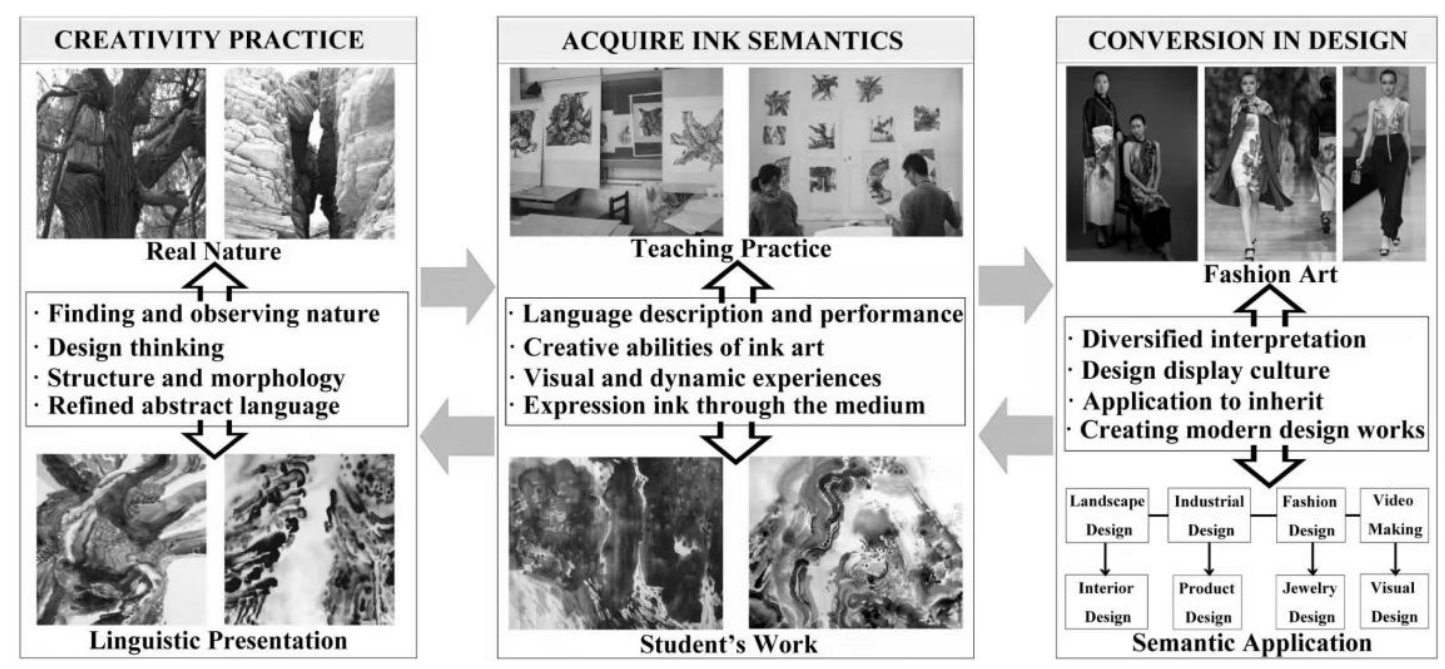

Figure 2. Model for ink semantic acquisition and conversion

\subsection{Conversion in Design}

In the practice of fashion creativity conversion, the symbols of ink culture are explored and refined from the artistic beauty, and the interpretation of meticulous multidimensional design is enlarged. The viewer is allowed to feel the artistic and cultural complex that modern design wants to convey, blend the oriental ink elements with modern design art, and show the designer's artistic thinking and claim.

- Ink in the conversion of fashion leaves blanks and space layout, and colouring and other methods of blank space are combined with the reconstruction of fabric media.

- The form aesthetic rules are used to deconstruct and reconstruct the ink effects in the design, and the spatial form of the morphological cognition method is combined with the creativity of the ink language to reflect the fashion elements and convey a certain theme and connotation.

- Clothing advanced customisation uses textile dyes and vegetable dyes. Combining traditional cultural elements with the new design themes enables the latter to become a vehicle for culture, to seek new support for traditional culture.

To guarantee the inheritance of traditional culture, it is necessary also to study and design the practice through many themes. Ink semantic acquisition and conversion becomes a valuable circulatory system.

\section{CONVERSION OF INK SEMANTICS}

When one talks about the integration of ink elements and design, one must consider the blending of aesthetics and application. Experimental ink is a kind of painting with strong characteristics, which conveys the visual design effect of imagery and artistic conception. This kind of imagery effect can produce rich imagination and conform to the aesthetic ideal of oriental art that incorporates artistic conception into design practice.

\subsection{Design Language: Imagery and Artistic Conception}

The imagery expresses the author's sentiment through the layout of the picture, often manifested by abstraction, and is also closely related to spirit, life, strength, and belief. In the oriental culture, human, art, and morality always act as a dynamic whole, and human emotions form an abstract material, thus conveying the artistic expressions of "nihility and existence growing out of one another" and 
"constructing the situation with emotion." In the creative process, "intention first then to write" is more attention to the emotion than the general skills.

Artistic conception is an important category of traditional Chinese aesthetics. It can be seen from this that artistic conception originally refers to the language of poetic space in which literary works blend the scenario and reality together. Artistic conception is the artistic realm of the blending of the subjective "idea" and the objective "environment." In the process of blending, rationality and emotion, form and spirit permeate each other to form the "artistic environment." The artistic concept can give people space for rich imagination, and it is a concrete manifestation of the Chinese traditional spiritual temperament and aesthetic taste.

The linguistic expression in design guides the designer to create a surreal artistic conception, such as ink art. The rich imagination and association make the works inspiring, guiding the viewers to see more artistic symbols beyond the surface and the things outside the picture, so that viewers can integrate into the artistic conception rendered by the design works. Therefore, in conveying the design, the designer needs to create an artistic realm by using the artistic elements, combine the works themselves with the theme ideas, show the artistic conception beyond the reality, and make the audience associate and produce rich characteristics and the implied ink art form.

\subsection{Design Language: Virtual and Real}

The ink element that can be perceived through vision is the artistic language of ink painting. This kind of artistic language emphasises the principle of expression of "virtual and real," which is the most important way to produce the artistic conception of design works. The so-called "virtual" refers to the parts of the ink painting that are light and implicitly vague in the forms of ink and water. The "virtual" part is usually the small part of the ink painting work or the blank part between the object and the pen. "Clearly" refers to the shape of the object with a strong and clear outline on the screen. In the linguistic expression of ink works, "the unity of the virtual and the real" can directly determine the existence of an artistic environment of ink integration design. Without virtual relational semantics, it does not become an artistic design, and there is no such thing as an artistic conception.

The "virtual" in ink painting can be used to represent the imaginary part of the design works. That is to say, in the practice of experimental ink painting, the blank part of the picture is not the useless part remaining on the composition, but the important artistic language of ink, which is another important factor that constitutes the beauty of the design form. In the design expression, despite the blank space, the viewer does not feel that it is a white paper, often empty and blank space is used to express a scene and feelings, and, through the association and imagination of people, it creates a certain artistic conception from the blank. It is like drawing or designing a fish - leaving white around it is reminiscent of water. If one is drawing or designing a bird, leaving white will be reminiscent of the blue sky. "Virtual" means leaving blank and space, leaving room for the viewer to fantasize. By letting the viewer enjoy the wonders of the outside world, the viewer can rely on the description of the design language to make it more artistic than the "real" object.

\section{Discussion}

The course is a process of continuous reflection in teaching. The use of cultural elements in the early curriculum is too simple and straightforward. The students' innovative thinking is limited by the image of the elements, and it is not enough to in-depth creativity in the later stages of design. It is unable to meet the needs of colleges and universities to train potential designers. The course tasks developed with experimental ink painting are active, and the uncertainty of the ink language unleashes the students' thinking. In practice, the design language is acquired by exploring the creativity of the artistic world, such as the shape, texture and quality of visual art, and the characteristics of ink. The experimental practice shows art and design with cultural heritage, giving the ink art and the contemporary design concept are mutually infiltrated, and to let the design works reflect the profound cultural charm. The formation of design semantics is the acquisition of individual design semantics produced by students in the commonality of educational practice. Achieve cultural individual expression through the commonality of knowledge in the design of educational courses.

The design works ultimately used by people, and the ink language conveys useful information about the design concept and cultural language to the user in the design transformation. The ink image of the culture is communicated in the process of realising the experience. Educators and students in product design, industrial design, fashion design, and engineering provide a source of practical creativity 
design. Regular artistic creation and self-expression in the classroom can produce a deep understanding of aesthetics. Teachers should have experience to guide students to sort out the rhyme of aesthetic categories from literary theory and design cases, and to deeply analyse its connotation, extension and perception. In practice, a higher level of design motivation can be observed among students, predicting possible solutions for a given design task and evaluating their design results. Furthermore, by implementing these measures at the origin of the creative process, the issue of insufficient creativity in later phases is solved. In particular, talent development should focus on shaping the core competitiveness, helping students to find the best self and focus on what they do best and what they need in the future.

\section{SUMMARY \& FUTURE WORK}

With the continuous development of art form, visual design, as one of the important forms of art design, is more and more important in the daily life and production of human beings. The design is not only a form of diversity but also a particularity of regional culture. The innovation of this study is that it addresses how to obtain an ink design language with oriental characteristics in the practice of creativity, and it can be a source of inspiration for designers in the process of design conversion. Creativity is both a process and a result. It is hoped that, when the accumulated method in a research field is applied to another field, real innovation will happen more frequently.

Design is a medium for expressing that should show the imprint of cultural heritage. It is important to learn some new design thinking by studying new ideas, cultures, and values [8]. The design work's use of cultural elements can not only create economic benefits, but also greatly promote the construction and innovation of human spiritual civilisation and culture heritage. Science and technology are constantly evolving, and we should reflect on the importance of cultural diversity in design. Design education training should be academically oriented to lead the cultural direction of the market. Based on the traditional culture and looking at the international perspective, it is necessary to train the young generation to carry forward its inheritance. In the future, the design of cultural language will be a technical system that realises the simultaneous development of technology product design. It is the linkage of all aspects of knowledge disciplines. It is multi-dimensional and needs to coordinate the cooperation of all parties to produce a new cultural design in a new era.

\section{ACKNOWLEDGMENTS}

The author presided over and was supported by China Ministry of Education Humanity and Social Science Research Youth Fund Project: Based on the nature of the spiritual core, a study on Han Chinese festival costumes culture form of inheritance (15YJC760022).

\section{REFERENCES}

[1] Grierson H. and Ion B. The concept of project memories: Improving the practice of global design information sharing. International Journal of Design Management and Professional Practice, 2013, 6(2), pp. 1-19. https://doi.org/10.18848/2325-162X/CGP/v06i02/38573

[2] Taura T. Introduction to an Interdisciplinary Approach. Creative Design Engineering, 2016, pp. 1-177. Elsevier Inc. https://doi.org/10.1016/C2015-0-01489-7

[3] Nagai Y., Taura T. and Mukai F. Concept blending and dissimilarity: factors for creative concept generation process. Design Studies, 2009, 30(6), pp. 648-675.

[4] Wang L. A Study of the Influence of Chinese Ink Painting Art and Calligraphy Art on Modern Graphic Design. In Lee, G (Ed.), 5th International Conference on Education and Education Management (EEM 2016), Vol. 92, pp. 425-428.

[5] Zhuang R. Study on Chinese painting freehand brushwork. In Lecture Notes in Electrical Engineering, 2013, Vol. 204 LNEE, pp. 639-645. https://doi.org/10.1007/978-1-4471-4802-9_84

[6] Bohemia E. Designing with and for others. Archives of Design Research, 2014, 112(4), 5. https://doi.org/10.15187/adr.2014.11.112.4.5

[7] McLening C. and Buck L. The effect of personality on the design team: lessons from industry for design education. International Conference on Engineering and Product Design Education, 2010, pp. 3-8.

[8] Nagai Y. and Taura T. Studies of Design Creativity: A Review and its Prospects. Journal of the Indian Institute of Science. Indian Institute of Science, 2015, October 1. 